\title{
Care begins when 9-1-1 is called: the evolving role of paramedic specialists in EMS Medical Communications Centres
}

\author{
Jan L. Jensen ${ }^{1}$. Andrew H. Travers ${ }^{2}$ - Alix J. E. Carter ${ }^{2}$
}

Received: 24 January 2022 / Accepted: 9 February 2022

(C) The Author(s), under exclusive licence to Canadian Association of Emergency Physicians (CAEP)/ Association Canadienne de Médecine d'Urgence (ACMU) 2022

When a patient calls 911, their pathway to emergency care begins. In most Canadian emergency medical services (EMS) systems, what ensues is a choreography of caller screening, triage, and paramedic deployment, followed by paramedic response, on-scene assessment, and care and most often transport to the emergency department (ED). Almost all Canadian EMS Medical Communications Centres (MCCs) are engineered to triage and dispatch resources, but not necessarily to provide patient care, or clinical support to responders. Several EMS systems in Canada and elsewhere are shifting away from the paradigm that all 911 calls require a paramedic response and/or ambulance transport to an ED. With the correct structures and processes, such as the MCC paramedic specialist teleconsultation role, there is opportunity to ensure that the right patient gets the right resource at the right time for the right reason.

In this issue of CJEM, Armour et al. present an interesting qualitative study exploring the emerging role of paramedic specialist online teleconsultant, in addition to traditional online medical oversight physician (OLMOP) [1]. This study explored the perceptions of British Columbia Emergency Health Services (EHS) paramedics on their clinical consults with paramedic specialists, compared to the traditional OLMOP consult. This study was novel and the findings are important for EMS systems considering adding an MCC paramedic specialist, as a few other publications describe such a role. The Nova Scotia EHS system has a similar program in which an advanced care paramedic works in the provincial EHS MCC, termed the Clinical Support Paramedic (CSP).

Jan L. Jensen

jan.jensen@emci.ca

1 Emergency Health Services/Emergency Medical Care, Dalhousie University, 239 Brownlow Avenue, Suite 300, Dartmouth, NS B3B2B2, Canada

2 Emergency Health Services, Nova Scotia Health, Dalhousie University, Dartmouth, NS, Canada
This role was in addition to traditional OLMOP consults. The CSP provides clinical support to paramedics for a prespecified set of call situations, rather than these calls going to OLMOP. We found a decrease in the number of calls which required EHS OLMOP consult with the introduction of the CSP role, and the relapse rate of non-transported patients was similar before and after the CSP implementation. Interestingly, the total number of requests for consultation increased after CSP implementation. These results give an indication that the advice provided by the CSP is as safe as that provided by OLMOP, and paramedics are supportive of this "phone a friend" option [2].

Most recently, in November 2021, EHS transitioned the OLMOP from on-call to a 24/7 on-site position in the MCC. This role, termed the Medical Communications Centre Physician (MCCP), works in close partnership with the CSP, medical communication officers, and operations leadership to provide more enhanced clinical support to front-line paramedics and to support patient flow and system navigation, such as this model of on-site physician decision support used in Ontario [3]. The evidence on the role of on-site physicians as part of a multi-disciplinary MCC team is limited but emerging [4]. This role along with an MCC nurse who can provide secondary triage of low acuity EMS callers and the paramedic specialist round out the concept of a robust, multi-disciplinary MCC clinical team.

Armour's finding that participants reported an increased number of consultations for non-mandatory topics is an indicator that the peer paramedic consultation service had also become culturally accepted among ground ambulance paramedics in the BC EHS system. If paramedics feel more comfortable asking their peers for non-mandatory advice, then this should we welcomed, as prehospital calls can be highly complex, as the authors noted in relation to the opioid crisis in the BC setting. As they say, 'when up poop creek, load the boat', or more eloquently stated, more heads are better than one. Currently, in a typical Canadian paramedic service, an OLMOP, sometimes referred to as "medical control", 
provides advice or direction to attending paramedic crews. Paramedics may be required by protocol to contact OLMOP or may elect to consult with them for clinical advice for various reasons, including to manage the clinical risk associated with some EMS calls, such as supporting transport decisions when patients are refusing transport or lack capacity to make such a decision, or to provide clinical approval to exceed or deviate from clinical protocols (such as medication dosing). As the paramedic profession continues to evolve, more options should be built into paramedic care guidelines and shift away from a "mother may I" approach towards more independent paramedic clinical practice. The paramedic specialist provision of peer clinical support to paramedics for clinical decision-making is a key aspect of this evolution. However, we note and agree with the gap identified by the authors that clinical governance and structured reportable outcomes are necessary to continue to advance the evidence base on the impact and effect of clinical paramedic specialists and to build the foundation needed to continue to safely add paramedic peer consult [5].

The paramedic specialist role also has potential to provide protective benefit to paramedics through real-time post-call debriefings. Paramedic specialists may be able to provide post-call clinical debrief feedback and real-time learning opportunities. Through these post-call consults, paramedic specialists may be in a unique position to identify the need for leadership, and peer or mental health supports for the ground ambulance paramedics, which potentially could reduce the risk of occupational stress injury.

In MCCs integrated with the larger health system, the paramedic specialist can play a much needed role in patient navigation through EMS-supported virtual care and telemedicine. For example, paramedic specialists can support improved diagnoses of certain conditions and trip destination decision-making, such as stroke or myocardial infarction cases. MCC paramedic specialists can also provide clinical support to front-line paramedics for treat-in-place cases, which may be of great value in COVID-19 situations, and serve a function in community paramedic programs. In Nova Scotia, this role serves an important function in clinical prioritization of inter-facility transfers to enable improved prioritization and patient flow.

The MCC of the future will likely evolve to include multi-disciplinary roles. EMS MCCs can and should go well beyond screening, triage, and dispatch, as care begins when 911 is called. There are relevant roles for a physician, nurse, and advanced level paramedic to provide more options for EMS callers and to provide robust clinical supports for responders and clinicians. The MCC paramedic specialist is a growing area of paramedic practice with future opportunities ripe for more research and evaluation.

\section{Declarations}

Conflict of interest The authors have no conflicts of interest to declare.

\section{References}

1. Armour R, Helmer J, Tallon J. Paramedic-delivered teleconsultations: a grounded theory study. CJEM. 2021. https://doi.org/10. 1007/s43678-021-00224-6.

2. Jensen JL, Al-Dhalaan F, Rose J, Carter AJE, McVey J, Butts F, et al. Ground ambulance paramedic clinical consults with a clinical support paramedic or nurse in an EMS communications centre compared to traditional EMS physician consults (abstract). Prehosp Emerg Care. 2015;19:174.

3. ORNGE. Annual Report 2020-21. Retrieved from: 2020-2021-Annual-Report-September-29_1.pdf (ornge.ca)

4. Langabeer JR, Gonzalez M, Alqusairi D, Champagen-Langabeer T, Jackson A, Mickahil J, Persse D. Telehealth-enabled emergency medical services program reduces ambulance transport to urban emergency departments. West J Emerg Med. 2016;17(6):713-20.

5. Jensen JL, Carter AJ, Rose J, Visintini S, Bourdon E, Brown R, McVey J, Travers AH. Alternatives to traditional EMS dispatch and transport: a scoping review of reported outcomes. CJEM. 2015;17(5):532-50. https://doi.org/10.1017/cem.2014.59 (Epub 2015 Mar 18 PMID: 26014661). 